\title{
Human Face Detection Technique using Haar-like Features
}

\author{
Srikanta Pal \\ Department of Computer Science and Application, \\ Swami Vivekananda Institute of Modern Science, Kolkata, India
}

\begin{abstract}
Research on biometrics has become most popular topic nowadays. Face is one of the most important biometric traits that plays a crucial role in our social association, passing on individuals' identity. Face detection is very important and challenging topic in the field of biometric research. It is also an essential step of any face recognition system. The actual benefits of face-based recognition or identification are uniqueness and acceptance. Face recognition or identification also has distinct advantages over other biometric systems because of its non-contact process while capturing the facial image. Face images are captured from a distance without any touch to the person who is being identified and the entire identification process does not require interacting with the person. Face recognition is a method of identifying people throughout facial images and it has many practical applications in the area of biometrics, information security, access control, smart cards, law enforcement and surveillance system. Therefore, face detection/recognition is one of the most interesting and important topics in research field. The goal of this study is to explore the face detection system using conventional face detection techniques using Haar-like feature and Haar-cascade classifier using OpenCv library. An encouraging average accuracy of $95.16 \%$ was achieved in this experiment.
\end{abstract}

\section{General Terms}

Document Image Processing, Computer Vision, Biometrics, Pattern Recognition.

\section{Keywords}

Haar-like features, face detection, cascade classifier, region of interest.

\section{INTRODUCTION}

Face detection as the keyword itself reveals it's meaning that it concerns about where a face is located in an image sample. In recent years, face recognition has attracted much attention and its research has rapidly expanded in computer vision and biometrics area, since it has many potential applications in computer vision communication and automatic access control system. Especially, face detection is an important part of face recognition as face detection is the first step of automatic face recognition technique. However, face detection is difficult enough because it has lots of dissimilarity of image appearance, such as pose variation (front, non-front), image orientation, occlusion, facial expression and illuminating condition, and many constraints like single face or multiple faces, image rotation, pose etc. So, there may occur some false detected regions of an image, which do not contain any face. In spite of all these problems there are lots of techniques available. Here, the study is started from the introduction of face detection and the areas where it is used.
Face recognition from image is a popular topic in biometrics research. Many community places usually have surveillance cameras for capturing video and these cameras have important value for security purpose. It is widely accepted that the face recognition have played a significant role in surveillance system as it doesn't require the object's cooperation. The real advantages of face-based recognition over other biometric traits are uniqueness and acceptance. As human face is a dynamic item having high degree of variability in its appearance, that makes face detection a challenging problem in computer vision field. In this area, accuracy and speed of identification is a main issue

Face recognition is a significant part of the capability of human perception scheme and is a routine task for individuals, while building a related computational model of face recognition. The computational model provides to theoretical perceptions and as well as many practical applications like access control, automated crowd surveillance, criminal identification, content based image database management, and so on.

The rest of this paper is written as follows. Related research works have been discussed in Section II. Haar-like feature detail is talked about in Section III. Section IV presents the integral image. Casecade classification technique is discussed in Section V. Database preparation is presented in Section VI. Section VII describes the experiment detail. The experimental results and discussion are presented in Section VIII. Error analysis is presented in Section IX. Finally, conclusions are presented in Section X.

\section{RELATED WORKS}

Selvi et al. [1] proposed a study that deals with face detection and recognition technique. The captured image from the web camera is detected first and then cropped it to feed to the system. This detection was performed through Viola-Jones Haar cascade classifier. Python-based Open CV software was used to detect the face and it was saved in Raspberry Pi. Once an image arrives, the server started to use LBPH algorithm on the face images. Finally their algorithm compares the emanated LBPH from detected image with the pre-saved $\mathrm{LBPH}$ in the database. The result of comparison was generated by sending a notification as authorized or unauthorized person via Internet of Things (IOT).

In a report, a Haar Cascade Classifier based approach for face recognition was introduced by Garg and Garg [2]. Their face recognition technique was based on the geometric features of a face which is probably the most intuitive approach to Human identification. The whole process was divided into three major steps where the first step is to find a good database of faces with multiple images for each individual. The next step is to detect faces in the database images and use them to train the face recognizer and the last step is to test the 
face recognizer to recognize faces.

In a study, a human face detection algorithm using three weak classifiers including Haar cascade classifier was introduced by Cuimei and Zhiliang [3]. Skin hue histogram, eye detection and mouth detection are the three classifiers adopted by this method. This yields sufficiently high detection. The proposed method generates a position prediction value (PPV) to about $78.18 \%-98.01 \%$. This can be amended to detect human faces only of multiple races and reduce the delay for detecting and recognizing various faces among different images of people with variation in light and background conditions.

In another report, Guennouni and Mansouri [4] implemented a face detection system by collating with Haar cascade classifiers and edge orientation matching. Edge orientation matching algorithm and Haar-like feature selection based cascade classifiers were the two techniques used in their system. Their algorithm produces a better matching performance but the detection speed was comparatively less.

\section{HAAR-LIKE FEATURES}

Haar-like features was developed by Viola and Jones on the basis of the proposal of Papageorgiou et. al [5]. The principle of Haar-like feature is based on detection of features encoding of some information about the object to be detected. It is basically applicable to classify generic objects. Haar-like feature is more particularly familiar for face recognition technique, where the system determines whether an object is a generic face or not. Technically, haar-like features refer to a way of slicing and dicing an image to identify the key patterns [6]. The template information is stored in a file known as a Haar-cascade, usually formatted as an XML file [7].

The calculation method of Haar-like features is faster by introducing integral image or summed area table [8]. For the reason, Haar-cascade and train-cascade classifiers compute features very quickly. The idea behind Haar-like feature selection algorithm is simple as described in [9]. It is based on the rule of computing the difference between the sum of white pixels and the sum of black pixels. The key advantage of this process is the fast computation of sum using the integral image. Haar-like is based on the same principle of Haar wavelets [10]. Haar-like features are adjacent rectangles in a particular position of an image. Types of Haar-like features depending on the number of adjacent rectangles are shown in Figure 1. According to Papageorgiou et al. [5] there are some types of Haar-like features. The value of a two-rectangle feature is the difference between the sums of the pixels within two rectangular regions. The regions have the same size and shape, and are horizontally or vertically adjacent (first line of Figure1). A three-rectangle feature computes the sum within two outside rectangles subtracted from the sum in a centre rectangle and this is represented as line feature (second line of Figure 1 ). A four-rectangle feature computes the difference between diagonal pairs of rectangles (lower left squares of third line of Figure 1 ). The last type of features is the centresurround feature (lower right squares of third line of Figure 1 ).

Generally, a Haar-like feature considers neighboring rectangular area at a specific location in a detection window. Then, the pixel intensities in each region are summed up and difference between these sums are calculated. This difference between the calculated sums is then used to categorize subsections of an image. Detection of human faces is one of the examples of this method. Commonly, the regions around the eyes are darker than the regions on the cheeks. One example of a Haar-like feature for face detection is therefore a set of two neighboring rectangular areas above the eye and cheek regions. [10]
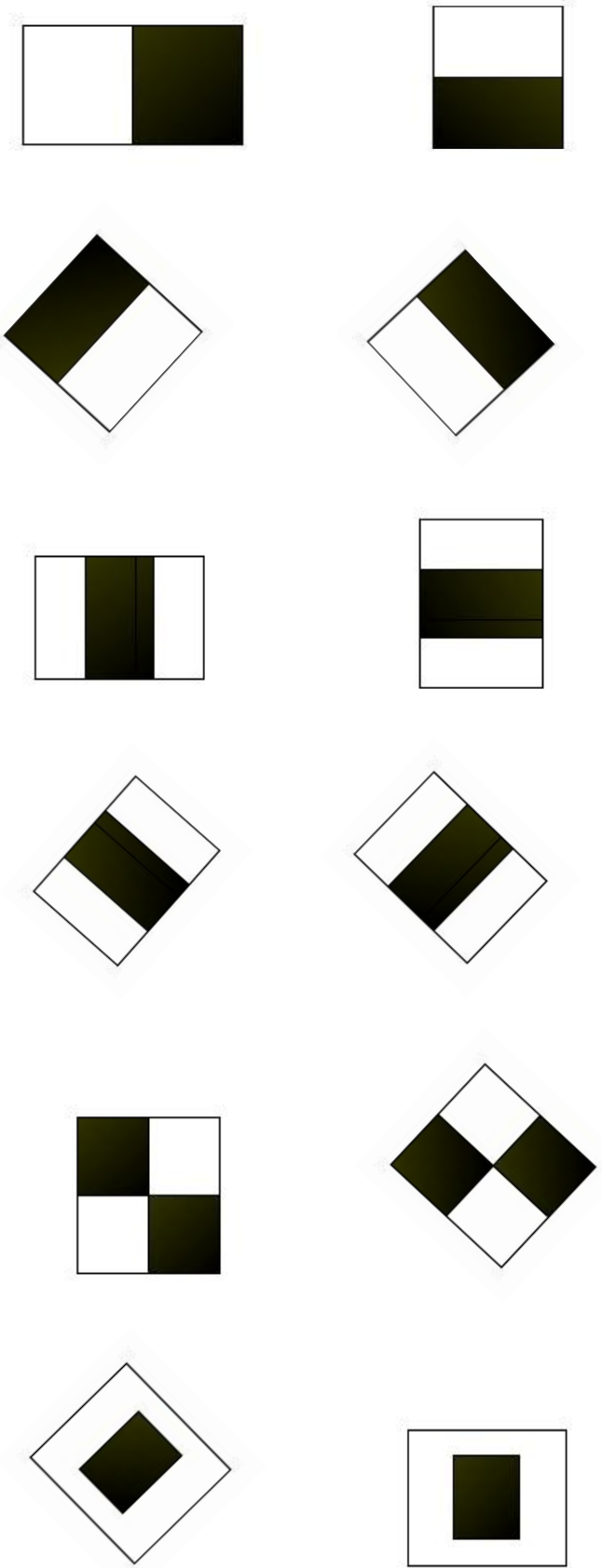

Fig 1. Simple haar-like features

\section{INTEGRAL IMAGE}

Rectangular two-dimensional features can be computed and extracted from an image sample quickly using an intermediate representation, is called the integral image [11]. The integral image, denoted by ii $(x, y)$, at location $(x, y)$ contains the sum of the pixel values which is on the above and to the left of ( $x$, $y)$. The integral image is outlined as: ii $[x, y]=i\left[x^{\prime}, y^{\prime}\right]$, where, $\mathrm{ii}[\mathrm{x}, \mathrm{y}]$ represents integral image, and $\mathrm{i}\left[\mathrm{x}^{\prime}, \mathrm{y}^{\prime}\right]$ represents original image.

Considering at the point $(x ; y)$, the value of the integral image is the sum of all the pixels above and to the left. Consider the following: 


$$
i i(x, y)=\sum_{x^{\prime} \leq x, y^{\prime} \leq y} \mathrm{i}\left(x^{\prime}, y^{\prime}\right)
$$

where $i i(x, y)$ is the integral image and $i\left(x^{\prime}, y^{\prime}\right)$ is the original image using the following pair of recurrences:

$$
\begin{aligned}
& s(x, y)=s(x, y-1)+i\left(x^{\prime}, y^{\prime}\right) \\
& i i(x, y)=i i(x-1, y)+s(x, y)
\end{aligned}
$$

The integral image is computed in one pass over the original image. The demonstration of the calculation method of summed area table is shown in Figure 2. For example by using only four array references, the sum of the pixels within rectangle $\mathrm{D}$ can be calculated according to the following ways:

at location 1 (sum of the pixels in rectangle A);

at location $2(\mathrm{~A}+\mathrm{B})$;

at location $3(\mathrm{~A}+\mathrm{C})$;

at location $4(\mathrm{~A}+\mathrm{B}+\mathrm{C}+\mathrm{D})$;

finally the sum within rectangle D is $4+1-(2+3)$.



Fig 2: Calculation of summed area table.

\section{CASCADE CLASSIFIER DETAILS}

It is a built-in package of python OpenCv[12], which supports only Haar-like features. By the word "cascade" it is indicated that the resultant classifier is consisting of a number of simpler classifiers that are applied subsequently to a region of interest.

It is a cascading of weak classifiers [13] used to boost the face detection process and reduce the computational complexity. Each node in the series contains a weak classifier and filter for one Haar feature. When a filter ignores to permit image regions, that specific sub window of the image is eliminated for further processing. It is then considered as a non-face, which means that the image regions that are processed do not contain the face to be detected. This is very imperative to the performance of the classifier, since all or nearly all negative image sub-windows will be eliminated in the first stage.

Conversely, when image regions successfully passed the filter, they go to the following stage, which contains a more complex filter. Only regions that successfully pass all filters are considered to contain a match of the face. This means that regions of the image contain the facial subject for detection. The reason behind the multi-stage classifier is to eliminate efficiently and rapidly the non-face sub-windows.

\section{DATABASE DETAILS}

Dataset is very important part for any experiment in research area. To conduct the experimentation of face detection technique a database is prepared. Preparation of database is always a difficult and time consuming task. A database consisting of 180 image samples are collected and prepared to conduct the experiment. Image samples are collected using a camera on mobile phone. Several number of human faces present in the captured image samples. The whole database is divided into six subsets based-on the number of the human faces presented in the photo frame. Each subset of database consists of 1 to 6 numbers of faces of people. The images also contain the body parts fully or partially with complex background. Images were captured arbitrarily without considering how complex is the background part. No photo capturing platform has been arranged for capturing the photos. Mainly the samples are collected from college campus, marriage halls, road sides, sea shore etc. So, different back ground pattern can be seen on the image samples. The experiment was conducted and the results are generated separately for each subset of face samples. Some image samples of the database are shown in Figure 3.
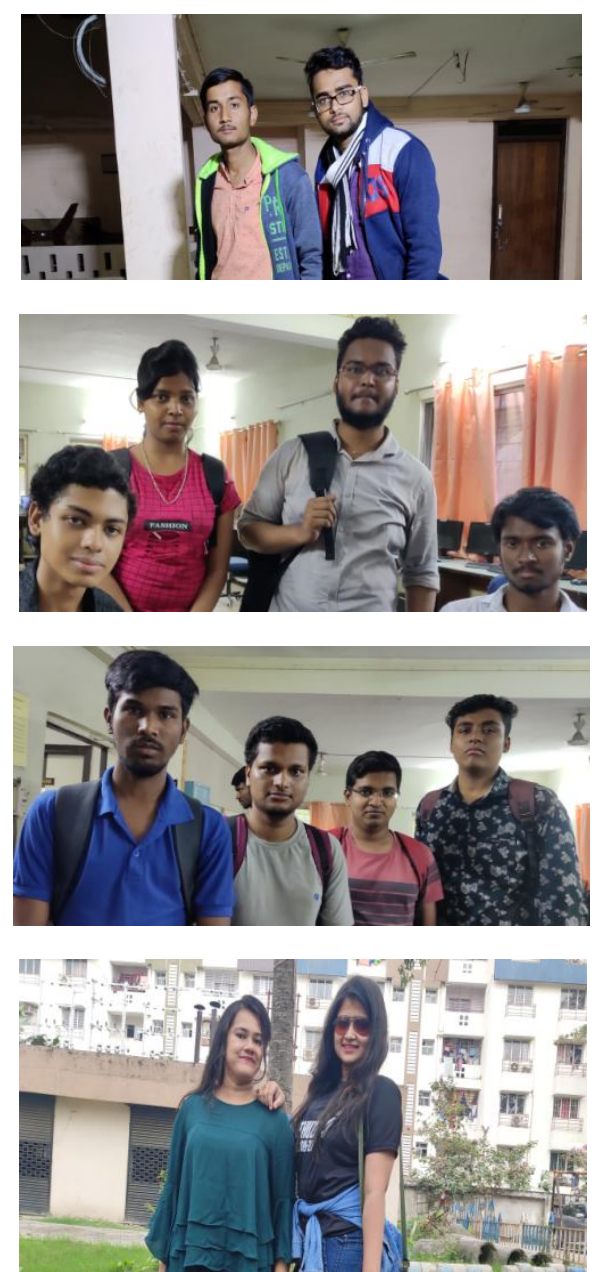

Fig 3. Some image samples of the database 


\section{EXPERIMENTS}

A conclusion section is not required. Although the open source library files are used for the proposed face detection technique using Haar-like feature and Cascade classifier. Mainly, python OpenCV library is considered for face detection method. When the system finds the faces it tries to detect the region of interest of the face positions in the image sample. If faces are found, it returns the region of interest positions of the detected faces as a function of $\operatorname{Rect}(\mathrm{x}, \mathrm{y}, \mathrm{w}, \mathrm{h})$, where $\mathrm{x}, \mathrm{y}, \mathrm{w}, \mathrm{h}$ are the co-ordinates of extreme corner points of region of interest of the face positions in an image sample. 'DetectMultiscale' module of python OpenCV is used also in this face detection technique. In order to achieve better system performance, two parameters of 'DetectMultiscale' module were tuned. Mainly these two parameters needed to be adjusted due to the number of faces presented in a single image sample. The parameters are scaleFactor and minNeighbors. i. scaleFactor: The value specifies how much the image size is reduced at each image scale. A lower value uses a smaller step for downscaling. This allows the algorithm to detect the face. It has a value of x.y, where $\mathrm{x}$ and $\mathrm{y}$ are arbitrary values and it can set. ii. minNeighbors: This parameter specifies numbers of 'neighbors' each candidate rectangle should have. A higher value results in less detections but it detects higher quality in an image.

\section{RESULT AND DISCUSSIONS}

The experiment was conducted separately for all the sub-sets of image sample. The result was also reported separately for different image sub-sets. As mentioned earlier in the Database Subsection that image samples were grouped into six based on the number of faces presented in a single image sample. Consequently, the accuracy achieved in the experimentation is provided in Table 1. Some image sample which is system generated by the system are shown in Figure 3. The face detection accuracy is presented in Figure 4. An encouraging highest accuracy of $98 \%$ was achieved in this experiment.

Table 1: Accuracy with different data sets

\begin{tabular}{|c|c|c|c|c|c|c|}
\hline Data Sets & Set-1 & Set-2 & Set-3 & Set-4 & Set-5 & Set-6 \\
\hline Accuracies & $99 \%$ & $98 \%$ & $96 \%$ & $96 \%$ & $92 \%$ & $90 \%$ \\
\hline
\end{tabular}



Fig 4. Representation of accuracies considering different sets of data.


Fig 5. Some images of system generated output.

\section{ERROR ANALYSIS}

Two system generated error samples have been shown in the Figure 5 and Figure 6 . The system is tested to see whether it can get face detection even when the face was obscured by a shade. The shaded faces were also detected by system properly. In Figure 5 and Figure 6 some faces are not detected by system. As shown in the Figure 6, the face which was not 
detected was also not fully exposed the front side. Some of the faces are very tiny. So, in these situations, the proposed technique fails to detect the faces.

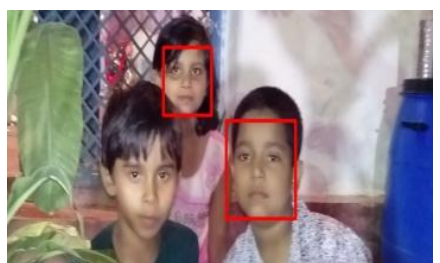

Fig 6. Error image sample with 3 faces

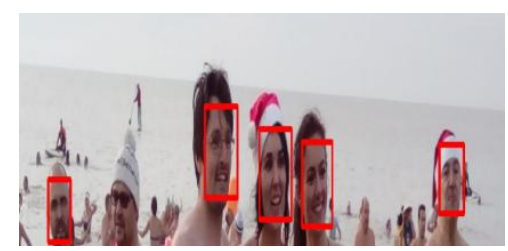

Fig 7. Error image sample with 6 faces

\section{CONCLUSIONS}

The approach of face detection method proffers an effective contribution to the field of biometric security. This study demonstrates an investigation of the performance of a face detection technique involving 180 image samples. The proposed approach used for the experimental process with Haar-like feature and Haar-cascade classifier using OpenCv library. The database, used for experimentation is divided into six subsets based-on the number of the human faces presented in the photo frame. Each subset of database consists of 1 to 6 numbers of faces of people. This face detection method minimizes computation time while achieving high detection accuracy. An encouraging accuracy of $95.16 \%$ is achieved in this experiment.

\section{REFERENCES}

[1] R Senthamizh Selvi , D. Sivakumar, J.S. Sandhya, S Siva Sowmiya, S Ramya, S Kanaga Suba Raja. "Face Recognition Using Haar - Cascade Classifier for Criminal Identification “, International Journal of Recent Technology and Engineering (IJRTE), volume-7, issue6S5, April 2019, pp. 1871-1876.

[2] Varun Garg and Kritika Garg, "Face Recognition Using Haar Cascade Classifier", Journal of Emerging Technologies and Innovative Research (JETIR), volume 3 , issue 12 , pp. 140-142.

[3] Li Cuimei, Qi Zhiliang, "Human face detection algorithm via Haar cascade classifier with three additional classifiers", International Conference on Electronic
Measurement \& Instruments, pp. 01-03, 2017.

[4] Souhail Guennouni and Anass Mansouri."Face Detection: Comparing Haar-like combined with Cascade Classifiers and Edge Orientation Matching", International Conference on Wireless Technologies, Embedded and Intelligent Systems (WITS), pp. 02-04, 2017.

[5] C. Papageorgiou, M. Oren, and T. Poggio. A general framework for object detection. In International Conference on Computer Vision, 1998.

[6] J. Shah, M. Sharif, M. Raza, and A. Azeem, "A survey: linear and nonlinear PCA based face recognition techniques," International Arab Journal of Information Technology, vol. 10, no. 6, pp. 536-545, 2013.

[7] C. Messom and A. Barczak, "Fast and efficient rotated haarlike features using rotated integral images," in Proceedings of the Australasian Conference on Robotics and Automation (ACRA '06), pp. 1-6, December 2006.

[8] P. Viola and M. Jones, "Rapid object detection using a boosted cascade of simple features," in Proceedings of the IEEE Computer Society Conference on Computer Vision and Pattern Recognition,pp. I511-I518, December 2001.

[9] S. Guennouni, A. Ahaitouf, and A. Mansouri, "Multiple object detection using OpenCV on an embedded platform," in Proceedings of the 3rd IEEE International Colloquium in Information Science and Technology (CIST '14), pp. 374-377, IEEE, Tetouan, Morocco, October 2014.

[10] R. Leinhart and J. Maydt, "An extended set of haar-like features for rapid object detection", Proceedings of the International Conference on Image Processing (ICIP '02), vol. 1, pp. I-900-I-903, 2002.

[11] F. C. Crow, "Summed-area tables for texture mapping", Proceedings of the 11th Annual Conference on Computer Graphics and Interactive Techniques (SIGGRAPH '84), pp. 207-212, 1984.

[12] Q. F. Zheng,W. Zeng, G.Wen, and W.Q.Wang, "Shapebased adult images detection," in Proceedings of the 3rd International Conference on Image andGraphics, pp. 150-153,December 2004.

[13] P. Viola and M. J. Jones, "Robust real-time face detection", International Journal of Computer Vision, vol.57, no.2, pp.137-154, 2004 